\title{
Ciencia de la sostenibilidad, sus características metodológicas y alcances en procesos de toma de decisiones
}

\author{
Sustainability science, its methodological \\ characteristics and reach in \\ decision-making processes
}

\section{Ciência da sustentabilidade, suas características metodológicas e alcance nos processos de tomada de decisão}

\author{
Walter Alfredo Salas-Zapata1 \& Leonardo Alberto Ríos-Osorio² \\ 'Bacteriólogo y Laboratorista Clínico. Doctor en Sostenibilidad, Tecnología y Humanismo. \\ ²Bacteriólogo y Laboratorista Clínico, Especialista en Parasitología Humana, \\ Doctor en Sostenibilidad, Tecnología y Humanismo. \\ ${ }^{1,2}$ Grupo de investigación Salud y Sostenibilidad. Escuela de Microbiología. \\ Universidad de Antioquia. Medellín, Colombia. \\ 1'wsalas@udea.edu.co. ${ }^{2}$ mleonardo@udea.edu.co
}

\begin{abstract}
Resumen
La transición hacia la sostenibilidad es el conjunto de cambios y decisiones que toma un grupo humano para resolver problemas de insostenibilidad. Para encarar estos problemas ha surgido una tendencia científica denominada ciencia de la sostenibilidad, la cual se ha considerado que debe ser una ciencia inspirada en el uso del conocimiento y debe tener características como la transdisciplinariedad, la participación, el aprendizaje social, la co-producción de conocimiento, la visualización de múltiples escalas y el manejo de la incertidumbre. No obstante, la investigación científica excepcionalmente permea procesos de toma de decisiones. Las posibilidades de lograrlo mejoran cuando las investigaciones posibilitan la relación y comunicación entre investigadores y tomadores de decisiones u otros actores. Este artículo analiza de qué manera las características en mención contribuyen a que las investigaciones
\end{abstract}

en sostenibilidad promuevan transiciones hacia la sostenibilidad.

Palabras Clave: metodología, participación, políticas, sostenibilidad, transdisciplinariedad

\section{Abstract}

The transition toward sustainability is the set of changes and decisions a group of people make in order to resolve problems of sustainability. To address these problems a scientific trend has emerged called sustainability science, which has been considered to be a science inspired by the use of knowledge and must have characteristics such as transdisciplinarity, participation, social learning, coproduction of knowledge, as well as consider multiple perspectives and scales of problems and the uncertainty inherent to them. However, scientific research plays a particularly 
important role in the decision making processes. The chances of success greatly increase when permits interaction and communication between researchers and decision makers, or other actor. This paper analyzes how the characteristics in question contribute to the promotion of changes toward sustainability.

Keywords: methodology, participation, policies, sustainability, transdisciplinarity.

\section{Resumo}

A transição para a sustentabilidade é o conjunto de mudanças e decisões de um grupo humano para resolver os problemas da insustentabilidade. Para resolver esses problemas surgiu uma tendência científica chamada ciência da sustentabilidade, a qual é considerada uma ciência inspirada no uso do conhecimento e deve ter características como a transdisciplinaridade, a participação, a aprendizagem social, a coprodução de conhecimento, a visualização multiescala e manejo de incerteza. No entanto, a pesquisa científica excepcionalmente permeia os processos de tomada de decisão. As possibilidades de sucesso melhoram quando as pesquisas permitem o relacionamento e a comunicação entre pesquisadores e tomadores de decisão e outros atores. Este artigo analisa como as características em questão contribuem para que as investigações de sustentabilidade promovam as transições para a sustentabilidade.

Palavras-chave: metodologia, participação, política, sustentabilidade, transdisciplinaridade.

\section{Introducción:}

Sostenibilidad es la capacidad que tiene un sistema de reajustar adaptativamente sus estructuras e interacciones socioecológicas para enfrentar las perturbaciones y persistir sin cambios significativos en sus atributos y funciones esenciales (Berkes, Colding, \& Folke, 2003; Folke, 2006; Holling, 1996, 2001; Norberg \& Cumming, 2008). Ello significa que un sistema es sostenible cuando los procesos y actividades humanas se adaptan a las características y dinámicas de los ecosistemas con los que se relacionan y a las características y necesidades socioeconómicas, culturales y políticas de los grupos humanos involucrados en tales procesos.

Así mismo, los problemas de insostenibilidad son situaciones que afectan las posibilidades de mantener el bienestar social y de los ecosistemas a causa de procesos humanos que tienen una escasa capacidad adaptativa a las dinámicas del entorno social y ecológico dentro de los cuales estos procesos se gestan y desarrollan.
Por esa razón, las transiciones hacia la sostenibilidad pueden entenderse como los cambios y decisiones desencadenados por los grupos humanos para resolver problemas de insostenibilidad.

Particularmente, en el último cuarto del siglo $X X$, el informe del Club de Roma (Meadows et al, 1972) y el informe Brundtland (WCED, 1987) reveló, tal vez, las primeras evidencias de problemas de insostenibilidad de carácter global. Entre estos problemas se pueden destacar el cambio climático, el agotamiento progresivo de fuentes de agua y de recursos energéticos, la deforestación, la creciente acumulación de desechos tóxicos, la contaminación de ríos y mares, y los desequilibrios en la acumulación de riqueza, entre otros.

Estos problemas tienen algunas características que los hacen diferentes a los problemas que comúnmente son objeto de estudio por parte de 
las ciencias clásicas. Primero, estos problemas siguen el comportamiento propio de los sistemas complejos. Esto significa que son fenómenos que emergen de múltiples causas y se manifiestan en diferentes escalas espaciales y temporales, poseen una incertidumbre irreducible, y la lógica de su solución no es la misma lógica que subyace a sus causas. Segundo, estos problemas son producto de procesos humanos que vinculan sistemas sociales y ecológicos pero no se adaptan a las dinámicas de éstos. Tercero, para resolver dichos problemas se requieren procesos de toma de decisiones relativamente urgentes que cambien las tendencias del comportamiento de estos, pues el mantenimiento de tales tendencias compromete las posibilidades de mantener el bienestar de un grupo humano dado y/o de los ecosistemas que sirven de soporte a ellos.

A este respecto, el informe del Club de Roma permitió cuestionar el tipo de desarrollo científico y tecnológico dominante en Occidente debido a los efectos inesperados que había traído consigo (Meadows, Meadows, Randers, \& Behrens, 1972:24) y a las limitaciones que tiene este modo de hacer ciencia para proponer soluciones a los problemas de insostenibilidad (Meadows, et al., 1972:27; Novo, 2006:7-22; Ravetz, 2006; Rios, Ortiz, \& Álvarez, 2009). En ese sentido, para promover transiciones hacia la sostenibilidad se hace necesario desarrollar un tipo de ciencia que permita concebir el carácter complejo de estos problemas y brinde luces en los procesos de toma de decisiones tendientes a resolverlos. De ahí el surgimiento de un programa de investigación denominado 'ciencia de la sostenibilidad'.

Se ha denominado 'ciencia de la sostenibilidad' a la tendencia que han tenido las comunidades científicas de orientar sus esfuerzos y programas de investigación al estudio del carácter dinámico de las interacciones naturaleza-sociedad para contribuir hacia la transición hacia la sostenibilidad (Clark \& Dickson, 2003; Kates et al., 2001). No obstante, la ciencia de la sostenibilidad no es aún una disciplina autónoma e independiente (Clark \&
Dickson, 2003) y no tiene un conjunto de principios propios para la construcción sistemática de conocimiento científico (Rapport, 2007). De igual modo, en este artículo se asume la ciencia de la sostenibilidad como la actividad científica que se desarrolla alrededor del estudio de la capacidad que tienen determinados sistemas de ajustar adaptativamente sus relaciones socioecológicas para sobreponerse a perturbaciones y mantener unos atributos y procesos esenciales.

Este escrito tiene por objetivo examinar las posibilidades que tiene la ciencia de la sostenibilidad de influir procesos de toma de decisiones a partir del análisis de los efectos prácticos que traería la aplicación de las características metodológicas sugeridas para este tipo de investigación.

\section{Relación entre investigación y procesos de toma de decisiones}

En términos generales, una transición hacia la sostenibilidad es un proceso de transformación social y de solución de problemas de insostenibilidad que puede lograrse como resultado de procesos de toma de decisiones formales e informales. Estas transformaciones obedecen a las decisiones que se gestan al interior de los grupos humanos ya sean en un marco políticoinstitucional o en un marco comunitario. Por esa razón entre los actores de estos grupos humanos pueden considerarse todas aquellas personas $u$ organizaciones que de uno u otro modo tienen alguna relación con el problema. De ese modo, al interior de los grupos se encuentran actores como los tomadores formales de decisiones, grupos de activistas, organizaciones sociales, instituciones, empresas, organizaciones comunitarias, sus representantes, líderes sociales, y personas de las comunidades que tienen algún vínculo con el problema.

La incorporación de conocimiento científico en procesos de toma de decisionesnoes un procesosenciIlo. Elconocimientogeneradoporlasinvestigaciones 
que es distribuido por las publicaciones científicas, tarda varios años en influir en la sociedad (Hertin et al., 2009). Cuando influye, en el contexto de los procesos de toma de decisiones, el conocimiento científico puede tener básicamente tres usos. Puede utilizarse para aprendizaje conceptual, que consiste en ilustrar a los tomadores de decisiones en el sentido de proveer información, ideas y perspectivas; aprendizaje instrumental, que se refiere a la utilización de éste para concretar decisiones; y puede tener un uso político, que es la utilización del conocimiento científico para lograr objetivos políticos y para justificar una decisión ya tomada (Hertin, et al., 2009). Estos tipos de utilización del conocimiento usualmente escapan al control de los investigadores.

En ese sentido, la actividad científica en general, y la investigación positivista en particular, ha recibido críticas por sus limitaciones para contribuir a los procesos de toma de decisiones. El contexto de las políticas de sostenibilidad no es la excepción (Espinosa, Harnden, \& Walker, 2008). Las críticas se deben a que la ciencia clásica ha asumido erróneamente que los resultados de las investigaciones serán utilizados racionalmente por los tomadores de decisiones cuando, contrario a ello, los conocimientos científicos no necesariamente son utilizados en la forma prevista por el investigador, ya que el uso de éstos depende fundamentalmente del contexto político, y en la mayoría de los casos, se utiliza para fortalecer posiciones políticas (Hertin, et al., 2009).

En muchos casos se ha visto que la investigación no produce el tipo de comprensión requerida por los hacedores de políticas; inclusive, a veces las preguntas científicas son muy cerradas y las escalas de trabajo son inconmensurables con las requeridas para las decisiones. También se ha visto que resulta más fácil incorporar conocimiento relevante en la fase inicial de una política pública que en sus fases posteriores; y de igual modo, para algunos científicos el problema con la utilización de la ciencia por parte de los hacedores de políticas es que ni escuchan ni entienden a los científicos y viceversa (GaIlopin, 2004).
Lo anterior no solamente se ha observado en el contexto de políticas de desarrollo sostenible. En el ámbito de otras políticas sectoriales, como las de salud, también se ha observado que las investigaciones tienen un papel secundario en el curso de las políticas y prácticas institucionales, pues las políticas no siempre requieren una investigación debido a que con frecuencia hay elementos de juicio suficientes para justificar una decisión determinada (Gómez-Arias, Orozco, Rodríguez, \& Velasquez, 2006); también se sabe que el conocimiento científico acumulado sobre un aspecto en particular no determina cambios significativos en el curso de una política (Almeida \& Bascolo, 2006); y además, la evidencia empírica señala que muchas decisiones políticas están basadas en consideraciones diferentes al conocimiento derivado de las investigaciones como, por ejemplo, las promesas de campaña y las prioridades de los partidos políticos (Fafard, 2008). Cabe resaltar que lo anterior no significa que la investigación positivista no sea necesaria sino que su alcance, para contribuir a procesos de toma de decisiones, es menor debido a su escasa capacidad de influir en procesos políticos y cambios en la conducta de los actores (Fafard, 2008; Torgerson, 2003).

En contraste con lo anterior, se ha demostrado que es más probable que la actividad científica influya directamente en procesos de toma de decisiones si durante las investigaciones se establecen canales de relación y comunicación entre los investigadores y los demás actores (GómezArias, et al., 2006). Estos canales son importantes porque permiten identificar los aspectos más relevantes de la relación investigación-decisión como lo son, la convergencia de intereses entre investigadores y hacedores de políticas; la presión que ejercen los contextos sociales, políticos e intelectuales; y la forma como unos se vinculan a los otros. Estos aspectos son relativamente más controlables por los investigadores que el uso de las publicaciones científicas, pues se presentan durante el proceso de investigación y no después de éste. 


\section{Características metodológicas para la investigación en sostenibilidad}

Para responder a la complejidad de los problemas de insostenibilidad y superar las limitaciones que tiene la ciencia positivista para promover soluciones, se han descrito diferentes características metodológicas que debe poseer la ciencia de la sostenibilidad. Una de ellas, sobre la que parece haber un consenso creciente, es la 'perspectiva de solución de problemas' (Boulanger \& Brechet, 2005; Fiksel, 2006; GaIlopín, 2001; Hirsch, Bradley, Pohl, Rist, \& Wiesmann, 2006; Kajikawa, 2008; Kates, et al., 2001; Kemp \& Martens, 2007; Komiyama \& Takeuchi, 2006; Luks \& Siebenhüner, 2007; Martens, 2006; Ravetz, 2006). Esta característica significa que la ciencia de la sostenibilidad no es ni ciencia básica ni aplicada sino una ciencia inspirada en el uso del conocimiento (Kajikawa, 2008) que procura brindar una comprensión integral de los problemas que investiga con el fin de alimentar procesos de deliberación tendientes a resolverlos (Kemp \& Martens, 2007) y de ese modo, que lleven a la implementación de prácticas que posibiliten la transición de grupos humanos hacia la sostenibilidad.

Así mismo, entre las otras características que también se han sugerido para la ciencia de la sostenibilidad (Tabla 1) se encuentran la transdisciplinariedad, la participación, el aprendizaje social, la co-producción de conocimiento, el abordaje de múltiples escalas y perspectivas, y la consideración de la incertidumbre inherente a los fenómenos (Boulanger \& Brechet, 2005; Fiksel, 2006; Funtowicz \& Ravetz, 1993; Gallopin, 2004; Gallopín, 2001; Grosskurth, 2007; Hirsch, et al., 2006; Kajikawa, 2008; Kates, et al., 2001; Kemp \& Martens, 2007; Komiyama \& Takeuchi, 2006; Luks \& Siebenhüner, 2007; Martens, 2006; Ravetz, 2006). Estas características dan cuenta de una marcada diferencia con respecto a la ciencia clásica dominante, que es esencialmente monodisciplinar, tecnocrática, y sustentada en la búsqueda de la predicción y de certidumbres sobre los fenómenos (Martens, 2006).

Tabla 1. Características metodológicas para la investigación en sostenibilidad

\begin{tabular}{ll}
\hline \multicolumn{1}{c}{ Característica } & Perspectiva \\
\hline Transdisciplinariedad & $\begin{array}{l}\text { Tecnocrática } \\
\text { Participativa }\end{array}$ \\
\hline Participación & $\begin{array}{l}\text { Activa } \\
\text { Pasiva }\end{array}$ \\
\hline Aprendizaje social & \\
\hline Co-producción de conocimiento & Temporales \\
Multiplicidad de escalas & Espaciales \\
& $\begin{array}{l}\text { Reducción de incertidumbre } \\
\text { cualitativa y cuantitativa }\end{array}$ \\
Manejo de incertidumbre & Manejo de incertidumbre irreducible \\
\hline
\end{tabular}




\section{Transdisciplinariedad}

La transdisciplinariedad es una característica metodológica que puede tener dos perspectivas, una tecnocrática y otra participativa. La transdisciplinariedad en sentido tecnocrático hace referencia a la integración disciplinar alrededor de un núcleo propio de conocimiento que es necesaria para responder a la complejidad propia de los problemas de insostenibilidad. Por lo tanto, aquí la incorporación de otras formas de conocimiento puede no darse o se dará según el carácter de las disciplinas que hagan parte de dicha integración. Por esa razón al aplicar un método con esta característica es muy posible que los actores afectados queden excluidos del proceso de investigación (Fiksel, 2006; Gallopin, 2004; Kajikawa, 2008; Komiyama \& Takeuchi, 2006).

En la transdisciplinariedad en sentido participativo, las disciplinas científicas se integran alrededor de núcleos propios de conocimiento y estos a su vez se integran con las visiones, valores, formas de conocimiento y normas sociales de los actores que no hacen parte del grupo de investigadores, alrededor de un problema (Gallopin, 2004; Hirsch, et al., 2006; Kemp \& Martens, 2007; Luks \& Siebenhüner, 2007). Esta acción integrada es un proceso de deliberación que permite conocer el problema en diferentes escalas y perspectivas, hacer explícitos valores y normas sociales en conflicto, identificar los actores más relevantes de las comunidades para la constitución de redes, comprender la complejidad de la realidad, mapear el rango de opciones de alternativas de solución, evaluar y discutir decisiones, establecer puntos y metas de referencia para la realización de actividades, informar los procesos de acción para el logro de ciertas consecuencias y explorar efectos no previstos de las decisiones y soluciones propuestas en circunstancias de incertidumbre.

La transdisciplinariedad participativa constituye por tanto un ejercicio dialéctico de cooperación entre diversos actores en el que se reconoce que el desarrollo de confianza y entendimiento mutuo brinda mucho potencial para las interacciones exitosas entre experticia y procesos democráticos (Kemp \& Martens, 2007) y ello depende, en parte, de la capacidad y voluntad que tenga una investigación de incorporar las perspectivas y preocupaciones de tales actores (Gallopin, 2004).

\section{Participación}

A grandes rasgos, existen dos formas de entender la participación: activa y pasiva. La participación activa se puede asumir como la construcción de conocimiento científico con los actores para comprender una realidad compleja (Grosskurth, 2007; Kemp \& Martens, 2007; Ravetz, 2006); mientras que la participación pasiva puede entenderse como la inclusión de la visión de los actores para determinar la aceptabilidad de las tesis científicas en situaciones de incertidumbre (Fiksel, 2006; Luks \& Siebenhüner, 2007), tal y como sucede en la ciencia posnormal (Funtowicz \& Ravetz, 1993).

Se puede decir que ambos tipos de participación contribuyen a mejorar las posibilidades de que una investigación influya en procesos de toma de decisiones públicas. No obstante, el hecho de que una investigación se lleve a cabo con participación pasiva supone que durante el proceso de investigación los actores están ausentes y, por lo tanto, son los investigadores quienes formulan el problema de investigación y deciden cuáles son las categorías de análisis más relevantes. Ello aumenta el riesgo de que las conclusiones de las investigaciones no correspondan a la complejidad del problema reflejada en los actores. Por esa razón la participación activa es más deseable cuando se pretende que una investigación contribuya a potenciar políticas o cambios sociales.

\section{Aprendizaje social}

Es la generación de cambios de actitudes y valores sociales en los actores durante el proceso de investigación debido a la adquisición de conocimientos que derivan en consecuencias prácticas (Hirsch, et al., 2006; Kates, et al., 2001; Kemp \& 
Martens, 2007; Luks \& Siebenhüner, 2007; Martens, 2006). La interacción entre los actores involucrados durante el proceso de investigación facilita la generación de aprendizaje social y de conocimiento más pertinente y utilizable en la solución de problemas (Luks \& Siebenhüner, 2007). Esta interacción y conocimiento produce transformaciones mutuas en las habilidades cognitivas y en las prácticas sociales, posibilitando a los actores, incluyendo los científicos, lograr una mejor comprensión de los problemas y de su realidad.

Cuando los sujetos comprenden su realidad y reconocen el problema como parte de esa realidad, su aprendizaje en el proceso de investigación hace que tomen mejores decisiones e implementen prácticas y acciones más apropiadas sin importar la capacidad predictiva del método de investigación empleado. Así, la imposibilidad de eliminar la incertidumbre de los procesos de toma de decisiones no se constituye en un obstáculo para una transición hacia la sostenibilidad debido a que el aprendizaje de los actores los faculta para decidir y actuar conforme a los cambios que sobrevengan con el tiempo.

El hecho de que el proceso de investigación dé lugar a un aprendizaje social implica que el acto de investigar hace más sostenible el sistema investigado en tanto la investigación misma es transformadora. En ese sentido es necesario tener una idea más clara de los procesos de aprendizaje que tienen lugar en la intersección entre el trabajo científico y el proceso político (Luks \& Siebenhüner, 2007).

\section{Co-producción de conocimiento}

La co-producción de conocimiento es un proceso interdependiente entre ciencia y política en el que simultáneamente se produce conocimiento y se genera orden social. La investigación científica genera conocimiento para alimentar los debates sobre la creación de normas institucionales que transforman la sociedad, mediante la creación de nuevos significados y representaciones del mundo político y social que, a su vez, generan identidad y legitimidad de la actividad científica Luks \& Siebenhüner, 2007.

\section{Multiplicidad de escalas}

Es la posibilidad que ofrecen las investigaciones de visualizar un problema en sus micro-macro niveles -escalas espaciales- (Boulanger \& Brechet, 2005; Gallopin, 2004; Gallopín, 2001; Martens, 2006), así como los ciclos completos de la dinámica de los fenómenos -escalas temporales- (Boulanger \& Brechet, 2005; Martens, 2006). Aquí, las dimensiones temporales y espaciales de los fenómenos pueden tener rangos tan amplios que en algunos casos la inclusión de los actores sería imposible. Como lo señala Martens (2006), para decir algo significativo de un fenómeno relacionado con el desarrollo sostenible sería necesario considerar dos generaciones o periodos de 25 a 30 años.

En estos casos los investigadores utilizan bases de datos y documentos que registran datos, valores, conocimientos y significados de los actores enmarcados en un momento histórico dado, pero ellos no necesariamente son quienes actúan directamente como fuente de conocimiento. El cambio climático y el crecimiento demográfico son solo dos ejemplos de problemas de investigación que, por su magnitud, requieren amplios rangos de observación y fuentes indirectas de conocimiento. En otros casos los rangos temporales y espaciales son tan cortos que es posible observar el ciclo completo de comportamiento de los fenómenos y sus manifestaciones simultáneas en los niveles local, regional y global a través de sus actores. Por ejemplo, la producción de biocombustibles es un fenómeno cuya dinámica completa puede ser observada en estos tres niveles.

\section{Manejo de incertidumbre}

Frente a la incertidumbre se pueden resaltar dos opciones: reducirla o encararla cuando es irreducible. La reducción de incertidumbre es la minimización 
de la incertidumbre que se tiene sobre los fenómenos mediante el mejoramiento del conocimiento y la comprensión de las propiedades emergentes y la magnitud de los mismos (Boulanger \& Brechet, 2005; Gallopin, 2004; Martens, 2006; Ravetz, 2006). Por esa razón puede tratarse de incertidumbre cualitativa o cuantitativa. En la reducción de la incertidumbre, la participación de otros actores es importante porque ellos pueden detectar relaciones entre los diferentes componentes de un fenómeno que los investigadores no pueden observar (Grosskurth, 2007). Así, las decisiones y acciones emprendidas contra los problemas reflejarán la complejidad expuesta por los actores, aunque su papel no será tan relevante en la medición de la magnitud de un fenómeno para reducir su incertidumbre.

El manejo de la incertidumbre irreducible es el conjunto de decisiones y acciones que se toman frente a situaciones sobre las que los conocimientos científicos tocan la frontera de lo cognoscible y no se puede reducir la incertidumbre (Boulanger \& Brechet, 2005; Funtowicz \& Ravetz, 1993; Ravetz, 2006). Esto sucede, por ejemplo, en el contexto del desarrollo tecnológico que suponen las plantas nucleares, donde se hace necesaria la participación de otros actores que cumplen el papel de ser tomadores de decisiones en la aceptación o no de vivir con dichas incertidumbres sobre los posibles impactos que puede traer dicha tecnología. En este caso los actores son una instancia de validación de las tesis científicas, pero no hacen parte del proceso de construcción de conocimiento científico, esto se ha denominado ciencia posnormal (Funtowicz \& Ravetz, 1993).

\section{¿Cuáles características metodológicas son más relevantes para influir en procesos de toma de decisiones?}

Las posibilidades que tiene la ciencia de la sostenibilidad de influir directamente, y no tardíamente, en la solución de los problemas de insostenibilidad depende de que la práctica de sus características metodológicas posibiliten durante el proceso de investigación el establecimiento de vínculos y de comunicación entre los investigadores y los demás actores relacionados con el problema.

En ese sentido, la transdisciplinariedad bajo una perspectiva tecnocrática no necesariamente permite la comunicación y los vínculos entre investigadores y otros actores. Ello no supone una subestimación de la transdisciplinariedad tecnocrática sino el reconocimiento de sus límites; pues la perspectiva tecnocrática asume que el conocimiento que resulta de las investigaciones será utilizado en las decisiones y políticas porque parte del supuesto de que estas decisiones son racionales. Pero contrario a ello se ha demostrado que la toma de decisiones no es un proceso racional y, por consiguiente, producir mejor conocimiento científico no significa que éste permeará las decisiones y acciones públicas (Almeida \& Bascolo, 2006; Gallopin, 2004; Gómez-Arias, et al., 2006).

La transdisciplinariedad participativa y el aprendizaje social dan cuenta de la integración de diferentes formas de conocimiento y ello necesariamente implica la participación de otros actores. En consecuencia, lo que permite que haya comunicación y vínculos entre investigadores y otros actores es la participación.

Así, la participación se convierte en una característica metodológica esencial. Sin ella no son posibles los vínculos y la comunicación entre investigadores y otros actores y, por tanto, sin ésta no sería posible la integración de diferentes formas de conocimiento de la realidad. La participación permite revelar y construir significados, incorporar los juicios de valor y las ideas colectivas vinculadas a los problemas de insostenibilidad, y construir definiciones normativas alrededor de la sostenibilidad del mundo. Por esa razón, desde el punto de vista metodológico la participación también significa una impregnación ética de la actividad científica en tanto supone un diálogo entre diferentes sistemas de valores. 
El aprendizaje social implica que durante el proceso de investigación los actores involucrados en el problema se ven inmersos en un proceso de aprendizaje. Éste influye directamente en las ideas, intereses y estructuras cognitivas de aquellos que son responsables de tomar decisiones o de implementar acciones prácticas.

Al igual que el aprendizaje social, la co-producción de conocimiento parece ser una característica que da cuenta de un propósito de la actividad científica que revela técnicamente la necesidad de la participación tanto de los científicos en instancias políticas como de otros actores en los procesos de investigación. Naturalmente esta característica implica participación y, por consiguiente, comunicación y vínculos entre investigadores y tomadores de decisiones.

Por otra parte, características como la multiplicidad de escalas y el manejo de incertidumbre no siempre requieren de la participación de otros actores. En el primer caso, a veces las escalas temporales y espaciales de un fenómeno pueden ser tan amplias que establecer contacto directo con los actores resultaría imposible para los investigadores. En el segundo caso, el manejo de la incertidumbre puede excluir actores cuando se intenta reducir la incertidumbre cuantitativa, pero puede incorporarlo si el problema requiere una reducción de incertidumbre cualitativa y/o goza de incertidumbre irreducible.
Al analizar la importancia de cada una de estas características, se puede decir que la transdisciplinariedad en sentido tecnocrático, la multiplicidad de escalas y el manejo de la incertidumbre pueden considerarse como características menos relevantes en términos de su contribución a la solución de problemas ya que su puesta en práctica no necesariamente implica la participación de los actores. En contraste, la transdisciplinariedad participativa, el aprendizaje social, y la co-producción de conocimiento implican la participación de los actores y permiten la comunicación y el establecimiento de relaciones entre estos y los investigadores. Sin embargo, entre estas características, la participación es el requisito indispensable para que las demás características se materialicen en la práctica y para que se viabilicen las relaciones entre investigadores y actores.

De ese modo se puede decir que en la búsqueda por lograr que las investigaciones influyan directamente en las decisiones y prácticas de los actores, existen unas características fundamentales como la participación, la transdisciplinariedad participativa, y el aprendizaje social y otras que adquieren menos relevancia como la co-producción de conocimiento, la reducción de incertidumbre cualitativa, la visualización de múltiples escalas y el manejo de incertidumbre irreducible (Figura 1).

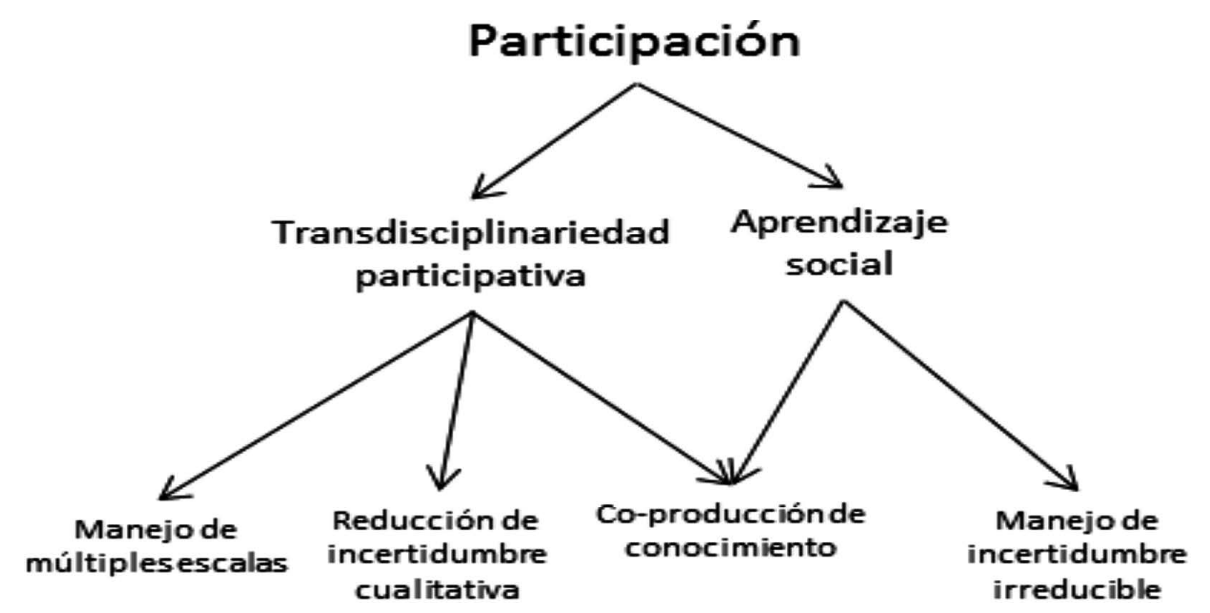

Figura 1. Relevancia de las características metodológicas en la transición hacia la sostenibilidad. 
Estas características llevan a los científicos a enfrentarse a una difícil tarea de comunicación, pues el mejoramiento de las habilidades comunicativas de los investigadores ayudaría en la generación de conciencia en los actores y el establecimiento de vías de consenso entre ellos. En ese orden de ideas, vale la pena preguntarse qué estilos comunicativos favorecen más la relación entre los investigadores y los actores sociales involucrados en el problema de interés.

\section{Conclusiones}

En contraste con las ciencias clásicas, la ciencia de la sostenibilidad se caracteriza por su afán de entender problemas complejos de insostenibilidad con el propósito explícito de contribuir a resolverlos. Por esa razón, además de ser una ciencia en la perspectiva de solución de problemas, la ciencia de la sostenibilidad implica un modo de hacer ciencia que es participativo y transdisciplinar, asume la incertidumbre propia de los fenómenos de la realidad y, en consecuencia, tiene un carácter más exploratorio y comprensivo que predictivo.

El conocimiento surgido de las investigaciones científicas no necesariamente influye en las prácticas y decisiones que toman los grupos humanos. Esta influencia depende de las prioridades políticas, de los compromisos de grupo, y de los intereses particulares de los actores. Por esa razón, las posibilidades de influir directamente en transformaciones sociales aumentan si la investigación permite la relación y comunicación entre investigadores y actores sociales.

La participación es una característica metodológica esencial para que las investigaciones en sostenibilidad contribuyan a favorecer transiciones hacia la misma. La puesta en práctica de esta característica permite a los investigadores y actores establecer vínculos y comunicación entre ellos. Por esa razón, además de la participación, otras características metodológicas de la ciencia de la sostenibilidad como la transdisciplinariedad participativa, el aprendizaje social, y la co-producción de conocimiento se convierten en las características más relevantes para contribuir a las transformaciones sociales, ya que la práctica de éstas requiere de manera inevitable de la participación de otros actores diferentes a los investigadores.

Cabe señalar que las características descritas aquí resultan de aplicar un criterio normativo, como es la perspectiva de solución de problemas, y que estas características no necesariamente tienen que estar presentes en todas las investigaciones en sostenibilidad. Ello dependerá de la naturaleza del problema del problema de investigación y de los propósitos mismos de las investigaciones.

\section{Literatura citada}

1. Almeida, C., \& Bascolo, E. (2006). Use of research results in policy decision-making, formulation, and implementation: a review of the literature. Cadernos de Saúde Pública, 22: (S7-19) (S20-33).

2. Berkes, F., Colding, J., \& Folke, C. (2003). Introduction. In F. J. C. C. F. Berkes (Ed.), Navigating social-ecological systems: Building resilience for complexity and change. Cambridge (UK): Cambridge University Press.

3. Boulanger, P., \& Brechet, T. (2005). Models for policymaking in sustainable development: The state of the art and perspectives for research. Ecological Economics, 55(3): 337-350.

4. Clark, W., \& Dickson, N. (2003). Sustainability science: the emerging research program. PNAS, 100 (14): 8059 - 8061.

5. Espinosa, A., Harnden, R., \& Walker, J. (2008). A complexity approach to sustainability - Stafford Beer revisited. European Journal Of Operational Research, 187 (2): 636-651.

6. Fafard, P. (2008). Evidence and Healthy Public Policy: Insights from Health and Political Sciences (Vol. Mayo). Quebec: National Collaborating Centre for Healthy Public Policy.

7. Fiksel, J. (2006). Sustainability and resilience: toward a systems approach. Sustain Sci Pract Policy, 2 (2): 14 - 21.

8. Folke, C. (2006). Resilience: the emergence of a perspective for social-ecological systems. Global Environ Chang, 16 (3): 253 - 267.

9. Funtowicz, S., \& Ravetz, J. (1993). Science for the postnormal age. Futures, 25 (7): 739-755.

10. Gallopin, G. (2004). Sustainable development: epistemological challenges to science and technology. Paper presented at the Workshop on Sustainable Development: Epistemological Challenges to Science and Technology, Santiago de Chile. 
11. Gallopín, G. (2001, December). Science and technology, sustainability and sustainable development. (LC/ R.2081/I). ECLAC.

12. Gómez-Arias, R., Orozco, D., Rodríguez, F., \& Velasquez, W. (2006). Políticas públicas en salud: relación entre investigación y decisión. Rev Fac Nac Salud Pública, 24 (2): 105-118.

13. Grosskurth, J. (2007). Ambition and reality in modeling: a case study on public planning for regional sustainability. Sustainability Sci Practice Policy, 3 (1): 3-11.

14. Hertin, J., Turnpenny, J., Jordan, A., Nilsson, M., Russel, D., \& Nykvist, B. (2009). Rationalising the policy mess? Ex ante policy assessment and the utilisation of knowledge in the policy process. Environment and Planning, 41 (5): 1185-1200.

15. Hirsch, G., Bradley, D., Pohl, C., Rist, S., \& Wiesmann, U. (2006). Implications of transdisciplinarity for sustainability research. Ecological Economics, 60 (1): 119-128.

16. Holling, C. (1996). Surprise for science, resilience for ecosystem, and incentives for people. Ecol Appl, 3: 733-735.

17. Holling, C. (2001). Understanding the complexity of economic, ecological, and social systems. Ecosystems, 4: 390 - 405

18. Kajikawa, Y. (2008). Research core and framework of sustainability science. Sustainability Science, 3 (2): 215-239.

19. Kates, R., Clark, W., Corell, R., Hall, J., Jaeger, C., Lowe, I., . . Svedin, U. (2001). Sustainability science. Science, 292 (5517): 641-642.

20. Kemp, R., \& Martens, P. (2007). Sustainable development: how to manage something that is subjective and never can be achieved? Sustain Sci Pract Policy, 3 (2): 5-14.
21. Komiyama, H., \& Takeuchi, K. (2006). Sustainability science: building a new discipline. Sustainability Science, 1 (1): 1-6.

22. Luks, F., \& Siebenhüner, B. (2007). Transdisciplinarity for social learning? The contribution of the German socio-ecological research initiative to sustainability governance. Ecological Economics, 63 (2-3): 418-426.

23. Martens, P. (2006). Sustainability: science or fiction? Sustainability Sci Practice Policy, 2 (1): 36 - 41.

24. Meadows, D. H., Meadows, D. L., Randers, J., \& Behrens, W. (1972). Los límites del crecimiento (Vol. Primera). Nueva York: Fondo de Cultura Económica.

25. Norberg, J., \& Cumming, G. (2008). Introduction. In J. Norberg \& G. Cumming (Eds.), Complexity theory for a sustainable future (pp. 1-14). New York: Columbia University Press.

26. Novo, M. (2006). El desarrollo sostenible. Su dimensión ambiental y educativa (First ed.). Madrid: PearsonPrentice Hall.

27. Rapport, D. (2007). Sustainability science: an ecolhealth perspective. Sustainability Science, 2 (1): 77-84.

28. Ravetz, J. (2006). Post-normal science and the complexity of transitions toward sustainability. Ecological Complexity, 3 (4): 275 - 284.

29. Rios, L., Ortiz, M., \& Álvarez, X. (2009). An epistemology for sustainability science: a proposal for the study of the health/disease phenomenon. International Journal of Sustainable Development \& World Ecology, 16 (1): 48-60.

30. Torgerson, D. (2003). Entre el conocimiento y la política: tres caras del análisis de política [1986]. In L. Aguilar (Ed.), El estudio de las políticas (pp. 197-238). México D.F.: Editorial Miguel Ángel Porrúa. 